\title{
Effects of mistuning on dynamic behavior of nonlinear cyclic systems with lump masses and cubic nonlinearity
}

\author{
E. Raeisi Estabragh ${ }^{1, a}$, S. Ziaei-RaD ${ }^{2}$ AND M. RAhimi ${ }^{3}$ \\ 1 Department of Mechanical Engineering, University of Jiroft, Jiroft, Iran \\ 2 Department of Mechanical Engineering, Isfahan University of Technology, Isfahan, Iran \\ 3 Departament de Matematica Aplicada III, LaC'aN, Universitat Polit'ecnica de Catalunya (UPC), 08034 Barcelona, Spain
}

Received 19 November 2014, Accepted 19 October 2015

\begin{abstract}
Mistuning in cyclic symmetric systems increases severely the forced response of system and splits the modes. This paper concerns with nonlinear behavior of mistuned cyclic systems. A nonlinear, mistuned model based on the method of multiple scales is proposed and formulated in which nonlinearity and mistuning parameter is assumed to be in of low order. Next, two mistuned systems were considered and solved by the multiple scale technique. Numerical results demonstrate that mistuning can lead to repeating and scattering of jump phenomena during the excitation frequency whereas in tuned cyclic system it occurs simultaneously (synchronously).
\end{abstract}

Key words: Nonlinear vibrations / bladed disk / mistuned system / multiple scale method

\section{Introduction}

Many unique and interesting phenomena occur in the dynamic response of nonlinear systems. Nayfeh, studied mathematically phenomenon such as jump that only can be observed in nonlinear systems [1]. Nayfeh in 1995 [1], Strogatz in 1994 [2], Verhulst in 1999 [3] and Rand in 2003 [4] studied the nonlinear oscillations. Also, localizing the nonlinear modes recently studied, this introduces the concept of nonlinear normal modes (NNMs) that in general relates to the synchronous and periodic study of nonlinear dynamical systems. The original theory for NNMs was conceived by Rosenberg in the early 1960s and may be considered as a direct extension of the concept of (real) normal modes of conservative linear systems [5]. Rosenberg defined NNM motion for a discrete system as a "vibration in unison", during which all coordinates oscillate with the same period, and reach their respective extreme simultaneously. Normal mode bifurcations have been investigated for discrete oscillators, where it has been shown that the number of modes for a nonlinear system may exceed the number of degrees of freedom [6]. Such a feature has no counterpart in linear theory. Papers published have shown that localized nonlinear normal modes exist for weakly coupled, nonlinear cyclic structures consisting of discrete oscillators [7], Moreover, investigations by King and Vakakis have demonstrated

\footnotetext{
a Corresponding author: ehsan1194@yahoo.com
}

the existence of nonlinear mode localization for flexible structures in the absence of structural disorder [8]. The works by Vakakis et al. offer overviews of the various methods of constructing nonlinear normal modes [9].

A nonlinear extension of the concept of mode shapes was proposed by Rand [10], Shaw and Pierre [11], Vakakis et al. [9] and Vakakis [12]. Weakly nonlinear systems were thoroughly analysed using perturbation theory [13-15]. Perturbation methods include for instance the method of averaging, the Lindstedt-Poincare' technique and the method of multiple scales and aim at obtaining asymptotically uniform approximations of the solutions. During the last decade or so, one has witnessed a transition from weakly nonlinear structures to strongly nonlinear structures (by strongly nonlinear systems, a system for which the nonlinear terms are the same order as the linear terms is meant) thanks to the extension of classical perturbation techniques $[16,17]$ and the development of new methodologies [18-21].

Several references have reported work on linear vibrations of cyclic systems $[22,23]$. Nonlinear vibrations of cyclic systems, including the response to standing and traveling wave (TW) excitation, nonlinear localized responses, and so on, have been considered in works by Vakakis, Bajaj, and other researchers [24, 25]. The dynamics of weakly coupled, nonlinear cyclic assemblies are investigated in the presence of weak structural mistuning. The method of multiple scales is used to obtain a set 
of nonlinear algebraic equations which govern the steady state, synchronous "modallike" motions for the structures [26].

The use of asymptotic techniques to find approximate solutions was addressed in several works; Vakakis et al. [29] used an averaging method, Gendelman et al. [28] used a multiple scale method, Mikhlin and Reshetnikova [29] used an expansion method in combining with a Mathieu equation comparison to investigate the stability of periodic solutions. Moreover Vakakis and Rand [30] proposed a method to derive exact solutions for systems with cubic nonlinearities based on the use of elliptic functions. Experimental results were also presented [31,32] in which the nonlinear device is made of a geometric nonlinearity.

Several authors, including Vakakis et al. [27] or Mikhlin and Reshetnikova [29] used the concept of Nonlinear Normal Modes (NNM) and their stability to explain this result. The concept of nonlinear normal modes was first introduced by Rosenberg [33] and has been the subject of many investigations in the past years. Several authors [9-11] demonstrated that the use of NNM in studying the dynamics of nonlinear (and in particular strongly nonlinear) systems has interesting applications both in free and forced responses.

One of the well-established analytical techniques for solving engineering vibration problems, which are represented by ordinary differential equations, is the method of multiple scales (MS). This method can be applied to find approximate solutions to a wide range of nonlinear problems. The main idea of the MS method is to split up the single independent variable into several new independent variables. The method allows the construction of a set of perturbation equations that can be solved under the condition of removal of secular terms. In order to analyze nonlinear vibrations of structural elements many have used the method of multiple scales which has been known to give a uniformly valid approximation as long as a specific system parameter is small.

One perturbation method that has received considerable attention for the NNM computation is the method of multiple scales [34-39].

In addition, nonlinear mode localization in periodically coupled oscillators due to nonlinear effects has also drawn increased attention $[40,41]$.

In the work by Samaranayake et al. a model of a weakly coupled multi degree-of-freedom cyclic system is studied for its primary resonance. The system possesses cubic nonlinearities, and the method of averaging is used to derive the amplitude or the averaged equations [42]. An approximate method to calculate the envelopes of the frequency response functions is developed in a study by Sextro et al. [43]. An example of a nonlinear system with cyclic symmetry is a bladed disk assembly with friction dampers. Regions where localization can occur with a high probability are calculated by this method.

Bladed disks may be used in several engineering systems such as fans, impeller pumps, turbine generators and jet engines. Ideally, these systems are tuned and all blades are identical but, in practice there always exist small, random differences among the blades due to manufacturing tolerances, in-operation wear, and so on that can make to destroy the cyclic symmetry of bladed disk. It is well known that even a small amount of mistuning can induce a large forced response known as mode localization and modal analysis shows that double eigenvalues appear in tuned cyclic symmetric structures which would be split in mistuned systems [22].

Judge et al. [44] have reported the results of an experimental investigation on the effects of random blade mistuning on the forced dynamic response of bladed disks. The primary aim of the experiment is to gain understanding of the phenomena of mode localization and forced response blade amplitude magnification in bladed disks.

Petrov et al. [45] formulated friction element under variable normal load conditions. The multiharmonic balance method (MHBM) is utilized for linearization of the nonlinearity to calculate the equivalent forcing vector as well as the tangent stiffness matrix.

An efficient method for analysis of nonlinear vibrations of mistuned bladed disk assemblies has been developed by Petrov and Ewins [46]. This development has facilitated the use of large-scale finite element models for realistic bladed disks, used hitherto in analysis of linear vibration, to be extended for the analysis of nonlinear multiharmonic vibration. Ciğeroğlu and Özgüven [47] developed a one-dimensional dynamic micro-slip element in which the inertial effects of the damper are included. The steady-state solution of the shear layer is determined by solving the nonlinear partial differential equations analytically.

Yan et al. [48] have used experimental mode analysis and mode correction to calculate some low-order modes of tuned blade and the disk with cone-flange. Beachkofski [49] used reduced order models to probabilistic rotor life assessment.

A damping strategy for bladed disks of turbomachinery involving a friction ring is investigated by Laxalde et al. [50]. Shin et al. [51] investigated the vibration localization phenomena due to mistuned coupling stiffness. Salhi et al. [52] present a methodology to identify the modal properties of mistuned bladed disk from tiptime data. Zhou et al. [53] present an adaptive control strategy based on passive piezoelectric shunt techniques for mistuned bladed disks. Resonant shunted piezoelectric patches are attached onto the disk between adjacent blades to reduce the blade vibration through blade-disk coupling. An essentially nonlinear piezoelectric shunt circuit is proposed by Zhou et al. [54] for the practical realization of nonlinear energy sink, and then applied to a mistuned bladed disk for blade vibration reduction.

In this paper, a formulation based on perturbation theory was used to find out the steady state solution of a cyclic system with nonlinear springs due to mistuning. The system parameters are considered to be lumped. The system is a simple representation of a real bladed disk system. Next, a multiple scale technique was employed for solving nonlinear equations. Finally, two cyclic systems 


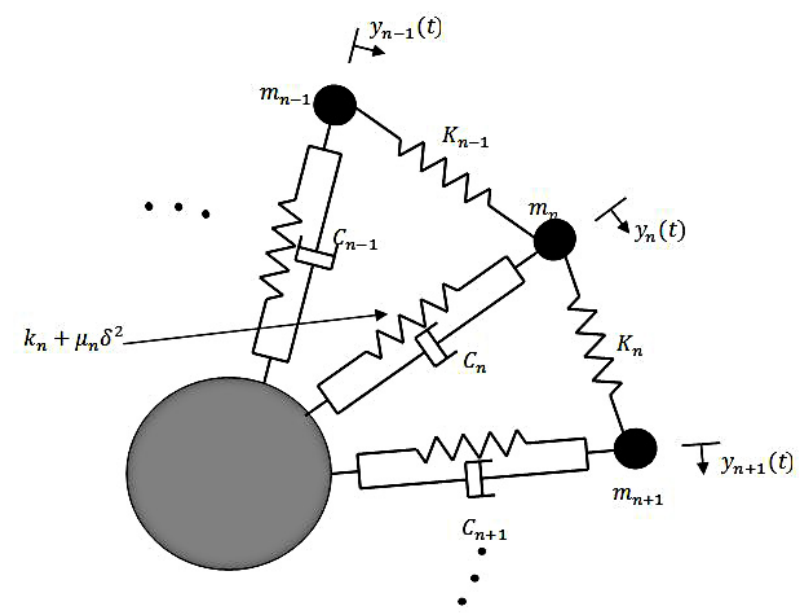

Fig. 1. Nonlinear cyclical symmetry systems dynamic model.

were considered and the tuned and mistuned responses of them were calculated and compared.

\section{Problem formulation}

The equation of motion for the $n$th pendulum of the model shown in Figure 1 can be written in the following form [1]:

$$
\begin{aligned}
m_{n} \ddot{y}_{n}+c_{n} \dot{y}_{n}+k_{n} y_{n}+K_{n}\left(y_{n}-y_{n+1}\right) & \\
+ & K_{n-1}\left(y_{n}-y_{n-1}\right)+\mu_{n} y_{n}^{3}=F_{n}
\end{aligned}
$$

where $m_{n}, c_{n}, k_{n}, \mu_{n}, K_{n}$ and $y_{n}$ denote the mass, damping coefficient, linear grounding stiffness and (cubic) nonlinear grounding stiffness, (linear) coupling stiffness connecting the $n$ and $n+1$ pendulum and displacement of the $n$th pendulum, respectively. For the cyclical symmetry based system following relations are true:

$$
y_{0} \equiv y_{N}, y_{N+1} \equiv y_{1}, \quad n=1, \ldots, N
$$

As it is clear dots means that the derivatives are with respect to the time. Here, we use the following change of variable for each non-dimensional equation (i.e., rescaling time with respect to the isolated (linear) natural frequency of the first pendulum):

$$
\tau=\sqrt{\frac{k_{1}}{m_{1}}} t
$$

By substituting Equation (3) into Equation (1), the following non-dimensional equation is obtained $[1,9]$ :

$$
\begin{gathered}
y_{n}^{\prime \prime}+\frac{c_{n}}{\sqrt{m_{1}} \sqrt{k_{1}}} \times \frac{m_{1}}{m_{n}} y_{n}^{\prime}+\frac{k_{n} m_{1}}{k_{1} m_{n}} y_{n}+\frac{K_{n} m_{1}}{k_{1} m_{n}}\left(y_{n}-y_{n+1}\right) \\
+\frac{K_{n-1} m_{1}}{k_{1} m_{n}}\left(y_{n}-y_{n-1}\right)+\frac{\mu_{n} m_{1}}{k_{1} m_{n}} y_{n}^{3}=\frac{m_{1} F_{n}}{k_{1} m_{n}}
\end{gathered}
$$

where the prime means derivatives with respect to $\tau$.
In this paper, the perturbation method for nonlinear problems is used. The external forces applied to each member are assumed to be harmonic with a small amplitude. The objective is to calculate the effects of small mistuning to cyclical symmetry of nonlinear systems. For this purpose, without lose of generality, a perturbation is considered in all physical parameters including the stiffness of spring, mass of each pendulum and damping coefficients. Assuming the properties of the first pendulum as parameters for nondimensionalization, the changes of physical parameters for other pendulum with respect to the first pendulum can be written as [1]:

$$
\begin{aligned}
\frac{k_{n}}{k_{1}} & =1+\varepsilon \hat{k}_{n}+O\left(\varepsilon^{2}\right), \quad \frac{m_{n}}{m_{1}}=1+\varepsilon \hat{m}_{n}+O\left(\varepsilon^{2}\right), \\
\frac{c_{n}}{\sqrt{m_{1}} \sqrt{k_{1}}} & =2 \xi \varepsilon+O\left(\varepsilon^{2}\right) \\
\frac{K_{n}}{k_{1}} & =\varepsilon \hat{K}+O\left(\varepsilon^{2}\right), \quad \frac{\mu_{n}}{k_{1}}=\hat{\mu}+O(\varepsilon), \\
\frac{F_{n}}{k_{1}} & =f_{n} \varepsilon^{\frac{3}{2}} \cos (\Omega \tau)+O\left(\varepsilon^{2}\right)
\end{aligned}
$$

where $\hat{k}_{n}$ and $\hat{m}_{n}$ are mistuning parameters corresponding to the stiffness and mass, respectively. Also $\varepsilon$ is a small dimensionless parameter and non-dimensional (where $|\varepsilon| \ll 1$ ). In Equation (5) mistuning, damping force, amplitude of harmonic force and coupling stiffness are assumed to be small. With using Taylor expansion for small $\varepsilon$ we have:

$$
\begin{aligned}
& \frac{2 \xi \varepsilon+O\left(\varepsilon^{2}\right)}{1+\varepsilon \hat{m}_{n}+O\left(\varepsilon^{2}\right)}=2 \xi \varepsilon+O\left(\varepsilon^{2}\right), \\
& \frac{1+\varepsilon \hat{k}_{n}+O\left(\varepsilon^{2}\right)}{1+\varepsilon \hat{m}_{n}+O\left(\varepsilon^{2}\right)}=1+\varepsilon\left(\hat{k}_{n}-\hat{m}_{n}\right)+O\left(\varepsilon^{2}\right) \\
& \frac{\varepsilon \hat{K}+O\left(\varepsilon^{2}\right)}{1+\varepsilon \hat{m}_{n}+O\left(\varepsilon^{2}\right)}=\varepsilon \hat{K}+O\left(\varepsilon^{2}\right), \\
& \frac{\hat{\mu}+O(\varepsilon)}{1+\varepsilon \hat{m}_{n}+O\left(\varepsilon^{2}\right)}=\hat{\mu}+O(\varepsilon)
\end{aligned}
$$

By substituting Equations (5) and (6) into Equation (4) and assuming small but finite oscillations $\left(y_{n}=\varepsilon^{1 / 2} x_{n}\right)$, one may obtain the following set of ordinary differential equations governing the forced response of the weakly coupled, weakly mistuned, nonlinear cyclic assembly:

$$
\begin{array}{r}
x_{n}^{\prime \prime}+2 \xi \varepsilon x_{n}^{\prime}+\left[1+\varepsilon\left(\hat{k}_{n}-\hat{m}_{n}\right)\right] x_{n}+\varepsilon \hat{K}\left(2 x_{n}-x_{n-1}-x_{n+1}\right) \\
+\varepsilon \hat{\mu} x_{n}^{3}+O\left(\varepsilon^{2}\right)=f_{n} \varepsilon \cos (\Omega \tau)
\end{array}
$$

where $n=1, \ldots, N$.

\section{Approximate solution using method of multiple scale}

One of the techniques for solving nonlinear equations is the method of multiple scale [1]. Multiple scale method 
is a global perturbation scheme that is useful in systems characterized by disparate time scales, such as weak dissipation in an oscillator. These effects could be insignificant on short time scales but become important on long time scales. Classical perturbation methods generally break down because of resonances that lead to what are called secular terms.

Let us first define independent time variables as [1]:

$$
T_{0}=\tau, \quad T_{1}=\varepsilon \tau, \quad T_{2}=\varepsilon^{2} \tau, \ldots
$$

Therefore, the $\frac{\mathrm{d}}{\mathrm{d} \tau}$ operator will take the following form:

$$
\begin{aligned}
\frac{\mathrm{d}}{\mathrm{d} \tau} & =\frac{\partial}{\partial T_{0}} \frac{\mathrm{d} T_{0}}{\mathrm{~d} \tau}+\frac{\partial}{\partial T_{1}} \frac{\mathrm{d} T_{1}}{\mathrm{~d} \tau}+\ldots \\
& =\frac{\partial}{\partial T_{0}}+\varepsilon \frac{\partial}{\partial T_{1}}+O\left(\varepsilon^{2}\right)=D_{0}+\varepsilon D_{1}+O\left(\varepsilon^{2}\right)
\end{aligned}
$$

Similarly, for the higher order derivatives we have:

$$
\frac{\mathrm{d}^{2}}{\mathrm{~d} \tau^{2}}=D_{0}^{2}+2 \varepsilon D_{0} D_{1}+O\left(\varepsilon^{2}\right)
$$

The response of Equation (7) is considered as [1]:

$$
\begin{aligned}
x_{n}(\tau, \varepsilon) & =x_{n}\left(T_{0}, T_{1}, \ldots\right)=\sum_{p=0}^{\infty} \varepsilon^{p} x_{n, p}\left(T_{0}, T_{1}, \ldots\right) \\
& =x_{n, 0}\left(T_{0}, T_{1}\right)+\varepsilon x_{n, 1}\left(T_{0}, T_{1}\right)+O\left(\varepsilon^{2}\right)
\end{aligned}
$$

By substituting Equations (9) to (11) into Equation (7), the following relations are obtained:

$$
\begin{gathered}
\left(D_{0}^{2}+2 \varepsilon D_{0} D_{1}\right)\left(x_{n, 0}\left(T_{0}, T_{1}\right)+\varepsilon x_{n, 1}\left(T_{0}, T_{1}\right)\right) \\
+2 \xi \varepsilon\left(D_{0}+\varepsilon D_{1}\right)\left(x_{n, 0}\left(T_{0}, T_{1}\right)+\varepsilon x_{n, 1}\left(T_{0}, T_{1}\right)\right) \\
+\left[1+\varepsilon\left(\hat{k}_{n}-\hat{m}_{n}\right)\right]\left(x_{n, 0}\left(T_{0}, T_{1}\right)+\varepsilon x_{n, 1}\left(T_{0}, T_{1}\right)\right) \\
+2 \varepsilon \hat{K}\left(x_{n, 0}\left(T_{0}, T_{1}\right)+\varepsilon x_{n, 1}\left(T_{0}, T_{1}\right)\right) \\
-\varepsilon \hat{K}\left(x_{n-1,0}\left(T_{0}, T_{1}\right)+\varepsilon x_{n-1,1}\left(T_{0}, T_{1}\right)\right) \\
-\varepsilon \hat{K}\left(x_{n+1,0}\left(T_{0}, T_{1}\right)+\varepsilon x_{n+1,1}\left(T_{0}, T_{1}\right)\right) \\
+\varepsilon \hat{\mu}\left(x_{n, 0}\left(T_{0}, T_{1}\right)+\varepsilon x_{n, 1}\left(T_{0}, T_{1}\right)\right)^{3}+O\left(\varepsilon^{2}\right)=f_{n} \varepsilon \cos (\Omega \tau)
\end{gathered}
$$

Equating the terms of different $\varepsilon$ order (coefficients $\left.\varepsilon^{0}, \varepsilon^{1}, \ldots\right)$ from both sides of Equation (12), the following equation is obtained for the largest scale (i.e $\varepsilon^{0}$ ):

$$
D_{0}^{2} x_{n, 0}\left(T_{0}, T_{1}\right)+x_{n, 0}\left(T_{0}, T_{1}\right)=0 \quad n=1, \ldots, N
$$

For terms of order $\left(\varepsilon^{1}\right)$, one can obtain:

$$
\begin{gathered}
D_{0}^{2} x_{n, 1}\left(T_{0}, T_{1}\right)+2 D_{0} D_{1} x_{n, 0}\left(T_{0}, T_{1}\right)+x_{n, 1}\left(T_{0}, T_{1}\right) \\
+2 \xi D_{0} x_{n, 0}\left(T_{0}, T_{1}\right)+\left(\hat{k}_{n}-\hat{m}_{n}\right) x_{n, 0}\left(T_{0}, T_{1}\right)+2 \hat{K} x_{n, 0}\left(T_{0}, T_{1}\right) \\
-\hat{K} x_{n-1,0}\left(T_{0}, T_{1}\right)-\hat{K} x_{n+1,0}\left(T_{0}, T_{1}\right)+\hat{\mu} x_{n, 0}^{3}\left(T_{0}, T_{1}\right) \\
=f_{n} \cos (\Omega \tau)
\end{gathered}
$$

Since higher order terms have small effect on the system response, it is sufficient to consider terms up to order of $\varepsilon^{0}$. For this purpose, the first response of Equation (12) is obtained for all components. Because of weak couplings between the components, the equation for each member can be separated and the response is:

$$
x_{n, 0}\left(T_{0}, T_{1}\right)=A_{n}\left(T_{1}\right) \mathrm{e}^{j T_{0}}+\bar{A}_{n}\left(T_{1}\right) \mathrm{e}^{-j T_{0}}
$$

where $j=\sqrt{-1}$. To obtain the second order response, we rewrite the external harmonic force as:

$$
f_{n} \cos (\Omega \tau)=\frac{f_{n}}{2}\left(\mathrm{e}^{j \Omega \tau}+\mathrm{e}^{-j \Omega \tau}\right)
$$

By substituting Equation (15) and Equation (16) into Equation (14), finally, it will transform to the following equation:

$$
\begin{aligned}
& D_{0}^{2} x_{n, 1}+x_{n, 1}=-2 j\left(D_{1} A_{n}+\xi A_{n}\right) \mathrm{e}^{j T_{0}} \\
& -\left(2 \hat{K}+\hat{k}_{n}-\hat{m}_{n}\right) A_{n} \mathrm{e}^{j T_{0}}+\hat{K} A_{n-1} \mathrm{e}^{j T_{0}}+\hat{K} A_{n+1} \mathrm{e}^{j T_{0}} \\
& \quad-\hat{\mu} A_{n}^{3} \mathrm{e}^{3 j T_{0}}-3 \hat{\mu} A_{n}^{2} \bar{A}_{n} \mathrm{e}^{j T_{0}}+\frac{f_{n}}{2} \mathrm{e}^{j \Omega \tau}+\text { C.C. } \quad \text { (17) }
\end{aligned}
$$

Without losing generality, we use the following assumption for the response analysis of the system around the resonance:

$$
\Omega=1+\sigma \varepsilon \rightarrow \Omega \tau=T_{0}+\sigma T_{1}
$$

where $\sigma$ is the detuning frequency which is the deviation from the natural frequency. By substituting Equation (18) into Equation (17), we obtain:

$$
\begin{gathered}
D_{0}^{2} x_{n, 1}+x_{n, 1}=\left[-2 j\left(D_{1} A_{n}+\xi A_{n}\right)-\left(2 \hat{K}+\hat{k}_{n}-\hat{m}_{n}\right) A_{n}\right. \\
\left.+\hat{K} A_{n-1}+\hat{K} A_{n+1}-3 \hat{\mu} A_{n}^{2} \bar{A}_{n}+\frac{f_{n}}{2} \mathrm{e}^{j \sigma T_{1}}\right] \\
\times \mathrm{e}^{j T_{0}}-\hat{\mu} A_{n}^{3} \mathrm{e}^{3 j T_{0}}+\text { C.C. }
\end{gathered}
$$

As we know, the coefficients of $\mathrm{e}^{j T_{0}}$ which are not zero, create secular terms which lead to infinite response. As a result, the coefficients of secular terms are set to zero.

$$
\begin{aligned}
-2 j\left(D_{1} A_{n}+\right. & \left.\xi A_{n}\right)-\left(2 \hat{K}+\hat{k}_{n}-\hat{m}_{n}\right) A_{n}+\hat{K} A_{n-1} \\
& +\hat{K} A_{n+1}-3 \hat{\mu} A_{n}^{2} \bar{A}_{n}+\frac{f_{n}}{2} \mathrm{e}^{j \sigma T_{1}}=0
\end{aligned}
$$

It is clear that Equation (20) is a first order nonlinear differential equation system with independent variable $T_{1}$ and dependent variables $A_{n}$, where $n=1, \ldots, N$.

The following general answer is proposed for Equation (20) in which coefficients $a_{n}$ and $\beta_{n}$ are functions of $T_{1}$ :

$$
A_{n}=\frac{a_{n}}{2} \mathrm{e}^{j \beta_{n}}
$$

By substituting Equation (21) into Equation (20), the following equation is obtained:

$$
\begin{aligned}
-2 j\left[\frac{a_{n}^{\prime}}{2} \mathrm{e}^{j \beta_{n}}+\frac{a_{n}}{2} \times j \times \beta_{n}^{\prime} \mathrm{e}^{j \beta_{n}}+\frac{a_{n}}{2} \times \xi \mathrm{e}^{j \beta_{n}}\right] \\
-\left(2 \hat{K}+\hat{k}_{n}-\hat{m}_{n}\right) \frac{a_{n}}{2} \mathrm{e}^{j \beta_{n}}+\hat{K} \frac{a_{n-1}}{2} \mathrm{e}^{j \beta_{n-1}} \\
+\hat{K} \frac{a_{n+1}}{2} \mathrm{e}^{j \beta_{n+1}}-3 \hat{\mu} \frac{a_{n}^{3}}{8} \mathrm{e}^{j \beta_{n}}+\frac{f_{n}}{2} \mathrm{e}^{j \sigma T_{1}}=0
\end{aligned}
$$


By multiplying Equation (22) in $\mathrm{e}^{-j \beta_{n}}$ we have:

$$
\begin{aligned}
&-j\left(a_{n}^{\prime}+a_{n} \times j \times \beta_{n}^{\prime}+\xi a_{n}\right)-\left(2 \hat{K}+\hat{k}_{n}-\hat{m}_{n}\right) \frac{a_{n}}{2} \\
&+\hat{K} \frac{a_{n-1}}{2} \mathrm{e}^{j\left(\beta_{n-1}-\beta_{n}\right)}+\hat{K} \frac{a_{n+1}}{2} \mathrm{e}^{j\left(\beta_{n+1}-\beta_{n}\right)}-3 \hat{\mu} \frac{a_{n}^{3}}{8} \\
&+\frac{f_{n}}{2} \mathrm{e}^{j\left(\sigma T_{1}-\beta_{n}\right)}=0
\end{aligned}
$$

And using the Euler equation $\left(\mathrm{e}^{j \alpha}=\cos (\alpha)+j \sin (\alpha)\right)$ :

$$
\begin{aligned}
& -j\left(a_{n}^{\prime}+\xi a_{n}\right)+a_{n} \beta_{n}^{\prime}-\left(2 \hat{K}+\hat{k}_{n}-\hat{m}_{n}\right) \frac{a_{n}}{2} \\
& +\hat{K} \frac{a_{n-1}}{2}\left[\cos \left(\beta_{n-1}-\beta_{n}\right)+j \sin \left(\beta_{n-1}-\beta_{n}\right)\right] \\
& +\hat{K} \frac{a_{n+1}}{2}\left[\cos \left(\beta_{n+1}-\beta_{n}\right)+j \sin \left(\beta_{n+1}-\beta_{n}\right)\right]-3 \hat{\mu} \frac{a_{n}^{3}}{8} \\
& \quad+\frac{f_{n}}{2}\left[\cos \left(\sigma T_{1}-\beta_{n}\right)+j \sin \left(\sigma T_{1}-\beta_{n}\right)\right]=0
\end{aligned}
$$

And then separating the complex equation into real and imaginary parts, the following equations are obtained:

$$
\begin{aligned}
& -\left(a_{n}^{\prime}+\xi a_{n}\right)+\hat{K} \frac{a_{n-1}}{2} \sin \left(\beta_{n-1}-\beta_{n}\right) \\
& +\hat{K} \frac{a_{n+1}}{2} \sin \left(\beta_{n+1}-\beta_{n}\right)+\frac{f_{n}}{2} \sin \left(\sigma T_{1}-\beta_{n}\right)=0
\end{aligned}
$$

$$
\begin{aligned}
& a_{n} \beta_{n}^{\prime}-\left(2 \hat{K}+\hat{k}_{n}-\hat{m}_{n}\right) \frac{a_{n}}{2}-3 \hat{\mu} \frac{a_{n}^{3}}{8}+\hat{K} \frac{a_{n-1}}{2} \cos \left(\beta_{n-1}-\beta_{n}\right) \\
& +\hat{K} \frac{a_{n+1}}{2} \cos \left(\beta_{n+1}-\beta_{n}\right)+\frac{f_{n}}{2} \cos \left(\sigma T_{1}-\beta_{n}\right)=0
\end{aligned}
$$

Equations (25) and (26) are a set of $2 n$ first order nonlinear differential equations with $2 n$ unknowns $\left(a_{n}\right.$ and $\left.\beta_{n}\right)$. After solving this set of equations by numerical methods, the final response for each member can be written as $x_{n}=\frac{a_{n}}{2} \mathrm{e}^{j\left(\beta_{n}+T_{0}\right)}+$ C.C. where for special condition it may represent a harmonic response.

To obtain the steady state response of Equations (25) and (26), the following change of variable is assumed:

$$
\gamma_{n}=\sigma T_{1}-\beta_{n}
$$

By substituting the above equation into Equations (25) and (26), the equations are rewritten in the form of:

$$
\begin{aligned}
a_{n}^{\prime} & =-\xi a_{n}+\hat{K} \frac{a_{n-1}}{2} \sin \left(\gamma_{n}-\gamma_{n-1}\right) \\
& +\hat{K} \frac{a_{n+1}}{2} \sin \left(\gamma_{n}-\gamma_{n+1}\right)+\frac{f_{n}}{2} \sin \left(\gamma_{n}\right) \\
a_{n} \gamma_{n}^{\prime}= & \sigma a_{n}-\left(2 \hat{K}+\hat{k}_{n}-\hat{m}_{n}\right) \frac{a_{n}}{2}-3 \hat{\mu} \frac{a_{n}^{3}}{8} \\
+ & \hat{K} \frac{a_{n-1}}{2} \cos \left(\gamma_{n}-\gamma_{n-1}\right)+\hat{K} \frac{a_{n+1}}{2} \cos \left(\gamma_{n}-\gamma_{n+1}\right) \\
+ & \frac{f_{n}}{2} \cos \left(\gamma_{n}\right)
\end{aligned}
$$

To have a steady state solution, the parameters $a_{n}^{\prime}$ and $\gamma_{n}^{\prime}$ are set to zero. As a result, we will have the following expression for the steady state response of nonlinear equations:

$$
x_{n}=a_{n} \cos \left(\Omega \tau-\gamma_{n}\right)
$$

Regarding to Equation (18), the above simplified equations are only valid for frequencies around the resonances. However, in nonlinear systems, the system response around the natural frequency is not maximum and the system may experience super and sub harmonic resonances.

\section{Numerical results}

In this section, two circular symmetric models were reviewed and the influence of the mistuning on the general properties of the steady state response was investigated.

\subsection{Three-degree-of-freedom system}

In order to create the random pattern, a new parameter was defined as:

$$
\hat{K}_{n}=2 \hat{K}+\hat{k}_{n}-\hat{m}_{n}
$$

By defining this parameter, it is possible to mistune all the components of the system simultaneously. Assuming the following values for the three-degree-of-freedom system:

$$
\begin{aligned}
& \varepsilon=10^{-3}, \xi=0.50, \hat{\mu}=25, \hat{K}=7, \hat{K}_{1}=7, \hat{K}_{2}=10, \\
& \hat{K}_{3}=16, \sigma=0 \rightarrow \Omega=1, f_{n}=1 n=1,2,3
\end{aligned}
$$

And then solving Equation (7) by direct integration technique, the blades response for the tuned and mistuned systems can be calculated. Figure 2 shows the blade response for the tuned and mistuned systems as a function of time.

For the case of 3DOFs system, the set of Equations (28) and (29) can be written in matrix form as:

\section{See equation (32) next page.}

Since the stimulating forces have the same phase, the three-degree-of-freedom tuned system responses are all exactly the same. Figure 3 shows the response amplitude, $a_{n}$ for all three tuned pendulums.

Next, the system mistuned under a random pattern. For the tuned system, it was assumed that $\hat{K}_{1}=\hat{K}_{2}=$ $\hat{K}_{3}=14$. Other parameters of the system are set as:

$$
\xi=0.50, \hat{\mu}=25, \hat{K}=7, f_{n}=1 n=1,2,3
$$

For the mistuned system, the mistuned parameters were $\hat{K}_{1}=7, \hat{K}_{2}=10, \hat{K}_{3}=16$. It is noteworthy that according to Equation (5), the mistuning parameters $\hat{K}, \hat{k}_{n}, \hat{m}_{n}$ and $\hat{K}_{n}$ all will multiply by $\varepsilon$. In other words, large difference between these values does not mean large changes in system physical parameters such as 


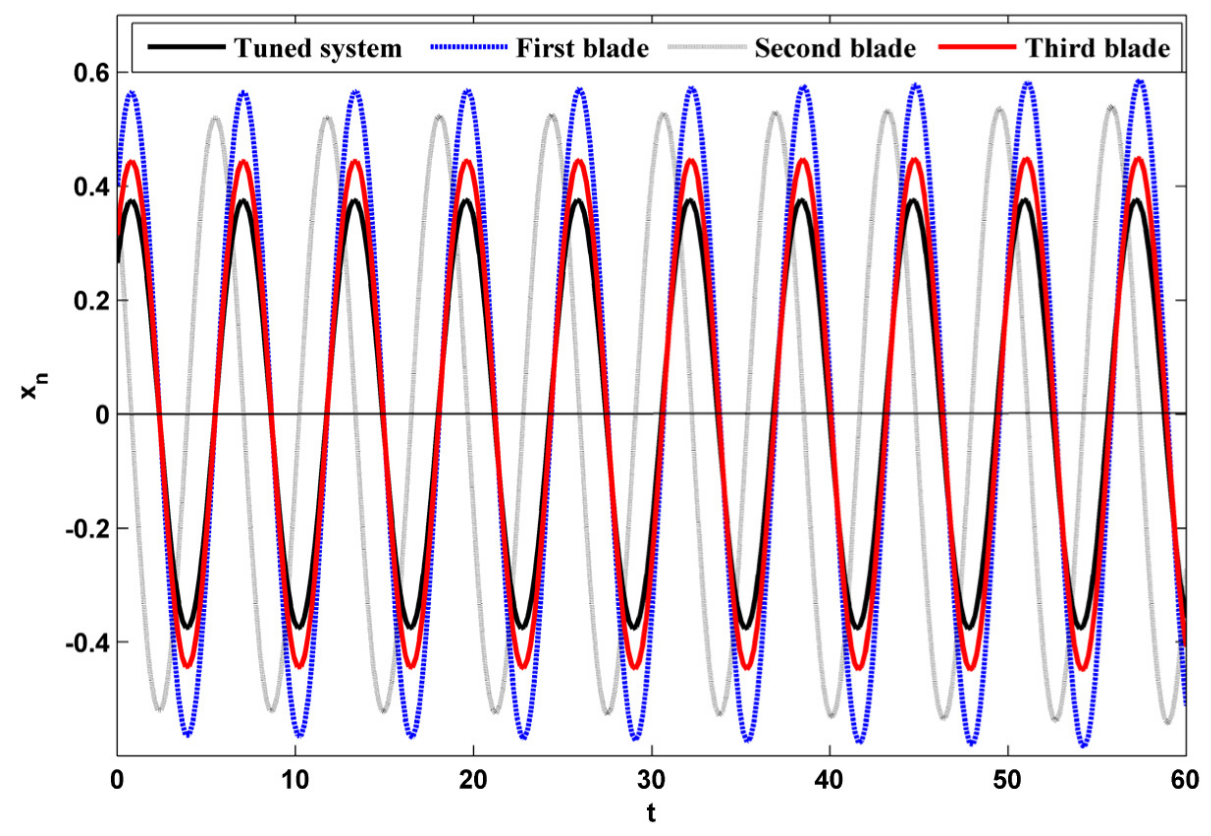

Fig. 2. Response amplitude, $x_{n}$, for all three mistuned pendulums versus $t$.

$\left[\begin{array}{c}a_{1}^{\prime} \\ a_{2}^{\prime} \\ a_{3}^{\prime} \\ \gamma_{1}^{\prime} \\ \gamma_{2}^{\prime} \\ \gamma_{3}^{\prime}\end{array}\right]=\left[\begin{array}{cccccc}-\xi & \frac{\hat{K}}{2} \sin \left(\gamma_{1}-\gamma_{2}\right) & \frac{\hat{K}}{2} \sin \left(\gamma_{1}-\gamma_{3}\right) & 0 & 0 & 0 \\ \frac{\hat{K}}{2} \sin \left(\gamma_{2}-\gamma_{1}\right) & -\xi & \frac{\hat{K}}{2} \sin \left(\gamma_{2}-\gamma_{3}\right) & 0 & 0 & 0 \\ \frac{\hat{K}}{2} \sin \left(\gamma_{3}-\gamma_{1}\right) & \frac{\hat{K}}{2} \sin \left(\gamma_{3}-\gamma_{2}\right) & -\xi & 0 & 0 & 0 \\ 0 & \frac{\hat{K}}{2 a_{1}} \cos \left(\gamma_{1}-\gamma_{2}\right) & \frac{\hat{K}}{2 a_{1}} \cos \left(\gamma_{1}-\gamma_{3}\right) & -\frac{3 \mu}{8} & 0 & 0 \\ \frac{\hat{K}}{2 a_{2}} \cos \left(\gamma_{2}-\gamma_{1}\right) & 0 & \frac{\hat{K}}{2 a_{2}} \cos \left(\gamma_{2}-\gamma_{3}\right) & 0 & -\frac{3 \mu}{8} & 0 \\ \frac{\hat{K}}{2 a_{3}} \cos \left(\gamma_{3}-\gamma_{1}\right) & \frac{\hat{K}}{2 a_{3}} \cos \left(\gamma_{3}-\gamma_{2}\right) & 0 & 0 & 0 & -\frac{3 \mu}{8}\end{array}\right]\left[\begin{array}{c}a_{1} \\ a_{2} \\ a_{3} \\ a_{1}^{2} \\ a_{2}^{2} \\ a_{3}^{2}\end{array}\right]$

$$
+\left[\begin{array}{ccc}
\frac{1}{2} \sin \gamma_{1} & 0 & 0 \\
0 & \frac{1}{2} \sin \gamma_{2} & 0 \\
0 & 0 & \frac{1}{2} \sin \gamma_{3} \\
\frac{1}{2 a_{1}} \cos \gamma_{1} & 0 & 0 \\
0 & \frac{1}{2 a_{2}} \cos \gamma_{2} & 0 \\
0 & 0 & \frac{1}{2 a_{3}} \cos \gamma_{3}
\end{array}\right]\left[\begin{array}{c}
f_{1} \\
f_{2} \\
f_{3}
\end{array}\right]+\left[\begin{array}{c}
0 \\
0 \\
0 \\
-\frac{\hat{K}_{1}}{2}+\sigma \\
-\frac{\hat{K}_{2}}{2}+\sigma \\
-\frac{\hat{K}_{3}}{2}+\sigma
\end{array}\right]
$$

$k_{1}, m_{1}, K_{1}, \ldots, k_{n}, m_{n}, K_{n}$. The calculated responses are depicted in Figure 4 for forward and backward motions. In this figure, the solid line graphs are the responses of the tuned system. It is clear that mistuning of the system have altered the response of each blade substantially. The responses of each blade experience three jump phenomena during forward or backward. Although the jump will happen for all blades in almost the same frequencies, the amplitude of vibration for each blade is different in all mistuning parameter range. The difference in vibration amplitudes mainly relate to the mistuning stiffness parameter $\hat{K}_{n}$.
The response amplitude of blades for both tuned and mistuned systems can be calculated from Figure 4 for case of $\sigma=0$. The maximum response of both tuned and mistuned systems can also be obtained from the responses depicted in Figure 2. These values are compared with each other in Table 1. Actually, the former is evaluated from the perturbation method while the latter is calculated by direct integration technique. As indicated in the table, the results obtained from both techniques are in good agreement. The maximum difference is less than $3.5 \%$.

Figure 5 shows the response of the 3 DOFs system for tuned and mistuned cases $(\hat{\mu}=0)$. As indicated in 
E. Raeisi Estabragh et al.: Mechanics \& Industry 17, 409 (2016)

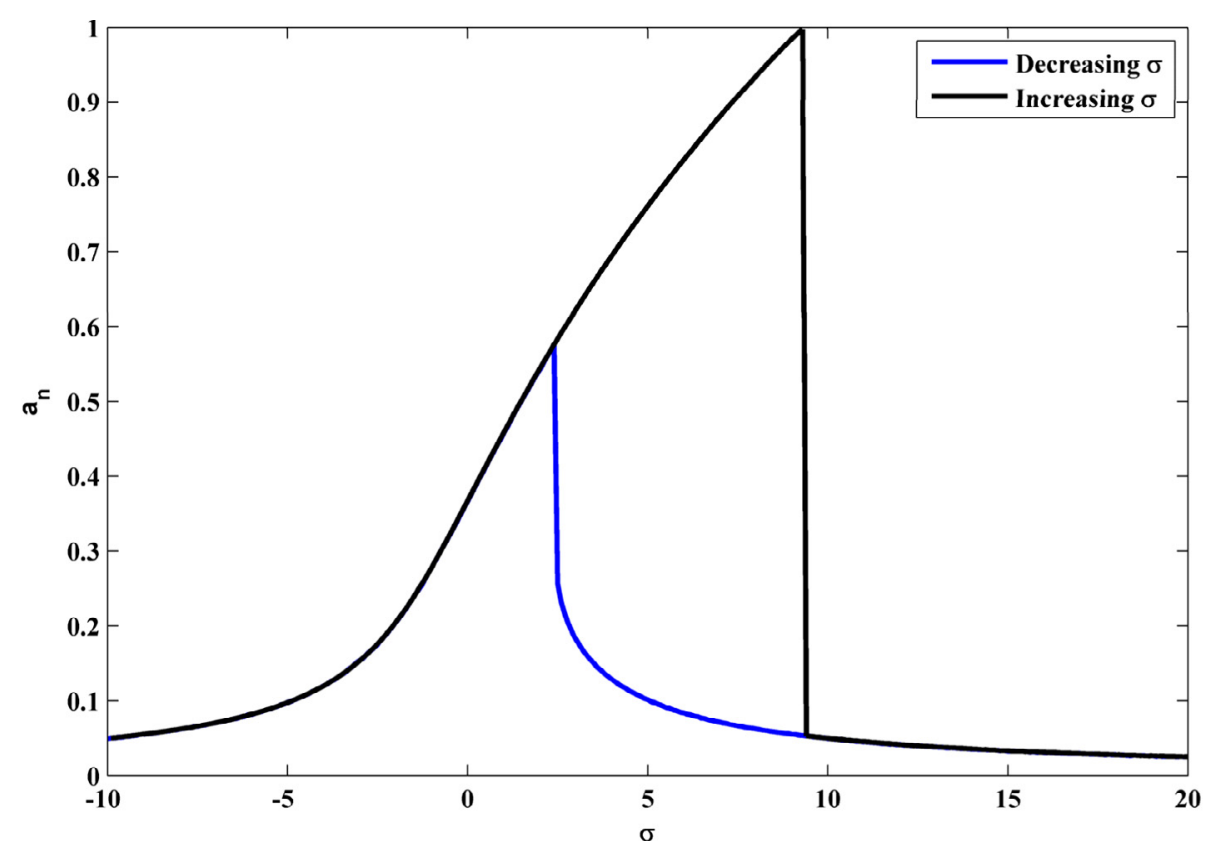

Fig. 3. Response amplitude, $a_{n}$, for all three tuned pendulums.

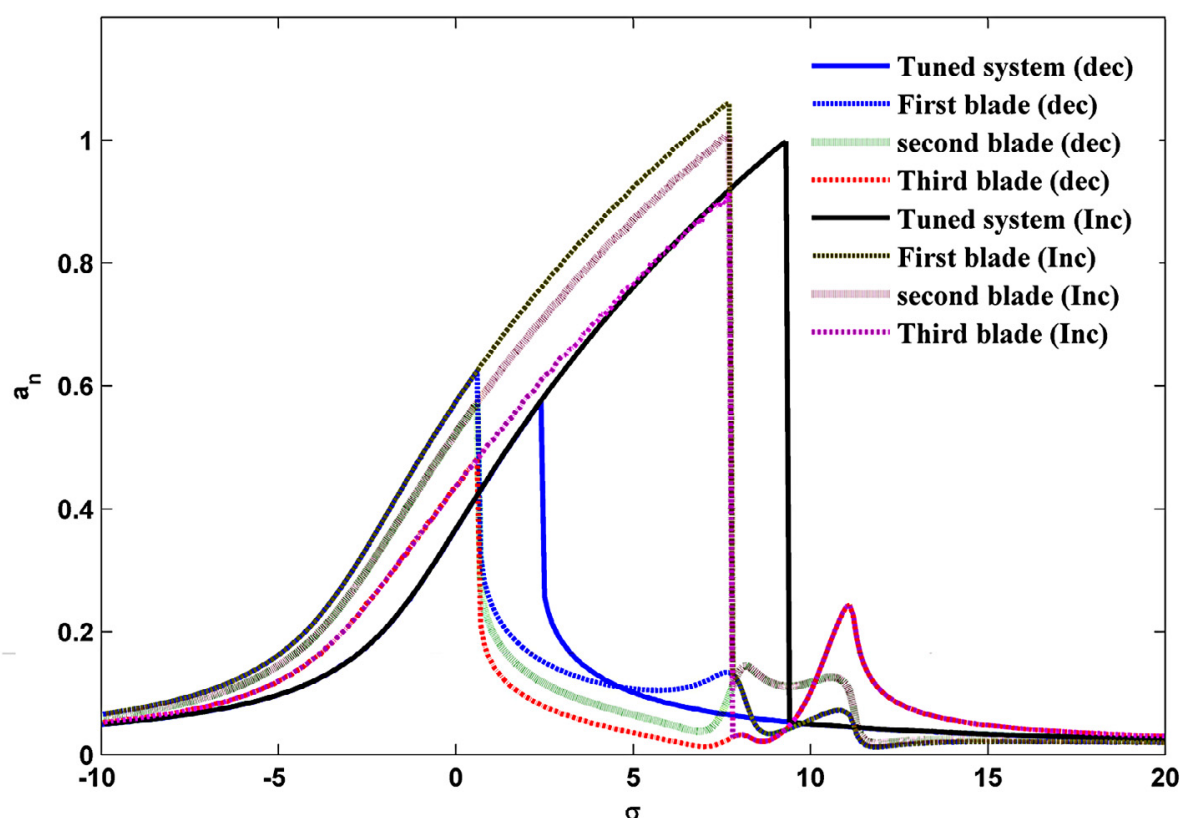

Fig. 4. Response amplitude, $a_{n}$, for all three mistuned pendulums.

Table 1. Comparison of direct integration and perturbation method.

\begin{tabular}{ccccc}
\hline System type & Amplitude & Direct integration & Perturbation method & \% Difference \\
\hline Tuned & $a_{n}$ & 0.3764 & 0.3677 & 2.3 \\
\hline \multirow{3}{*}{ Mistuned } & $a_{1}$ & 0.5868 & 0.5743 & 2.1 \\
& $a_{2}$ & 0.5408 & 0.5235 & 3.2 \\
& $a_{3}$ & 0.4492 & 0.4371 & 2.7 \\
\hline
\end{tabular}




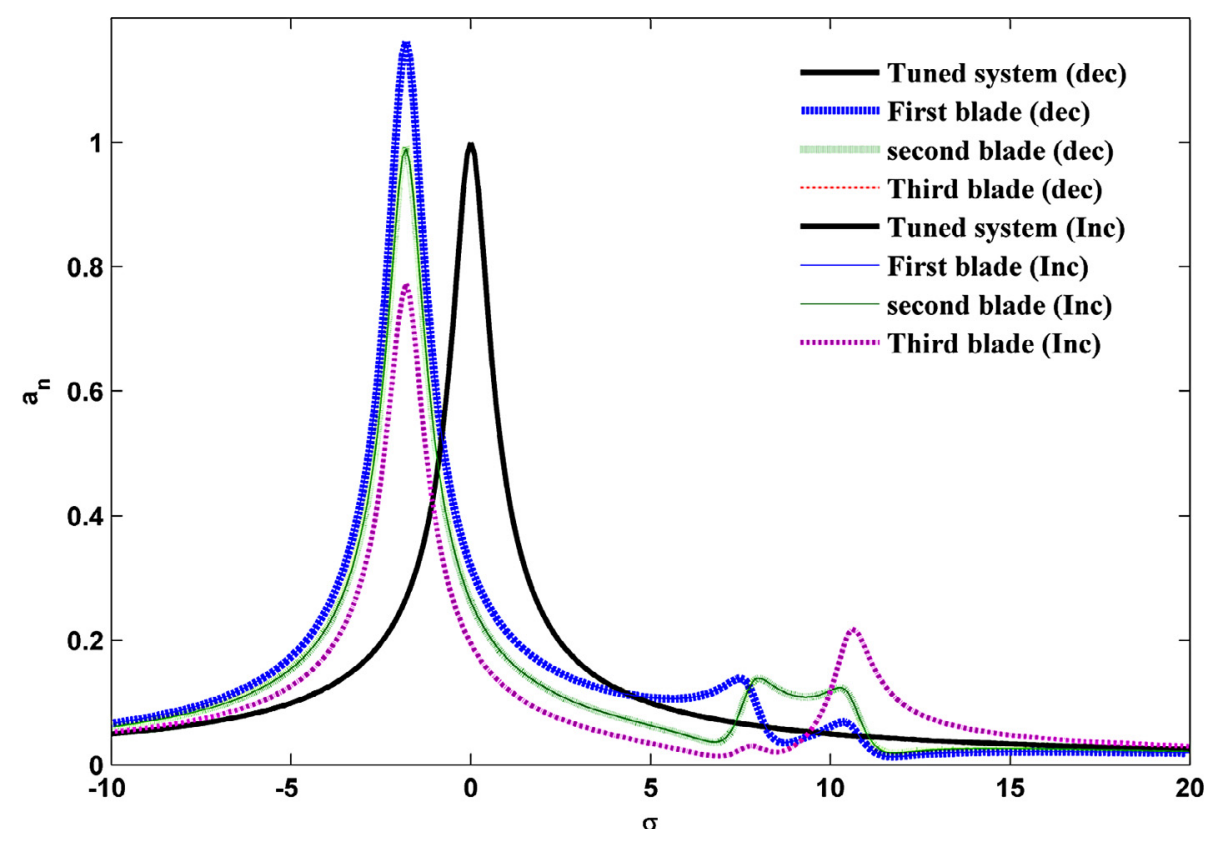

Fig. 5. Response amplitude, $a_{n}$, for all three mistuned pendulums (for $\hat{\mu}=0$ ).

Table 2. Maximum response amplitude $a_{n}$, for all three pendulums.

\begin{tabular}{|c|c|c|c|c|c|c|}
\hline System type & $\hat{\mu}$ & $\sigma$ & $\operatorname{Max} a_{1}$ & $\operatorname{Max} a_{2}$ & $\operatorname{Max} a_{3}$ & $\operatorname{Max} a_{n}$ \\
\hline \multirow{4}{*}{ Tuned } & \multirow{2}{*}{$\hat{\mu}=0$} & increase & 1 & 1 & 1 & 1 \\
\hline & & decrease & 1 & 1 & 1 & 1 \\
\hline & \multirow[b]{2}{*}{$\hat{\mu}=25$} & increase & 0.9978 & 0.9978 & 0.9978 & 0.9978 \\
\hline & & decrease & 0.5766 & 0.5766 & 0.5766 & 0.5766 \\
\hline \multirow{4}{*}{ Mistuned } & \multirow{2}{*}{$\hat{\mu}=0$} & increase & 1.1587 & 0.9892 & 0.7735 & 1.1587 \\
\hline & & decrease & 1.1587 & 0.9892 & 0.7735 & 1.1587 \\
\hline & \multirow{2}{*}{$\hat{\mu}=25$} & increase & 1.0593 & 1.0083 & 0.9137 & 1.0593 \\
\hline & & decrease & 0.6246 & 0.5723 & 0.4825 & 0.6246 \\
\hline
\end{tabular}

the figure the location and the amplitude of the maximum response of the mistuned system have changed in comparison with the tuned system.

The maximum response of the blades for different scenarios is given in Table 2. As indicated in the table, the maximum response for the mistuned case is larger than the tuned one. In tuned case, the response of all three blades is the same. For $\hat{\mu}=0$, the maximum response of all blades is equal to unity for either increasing or decreasing the vibration parameter $\sigma$. For $\hat{\mu}=25$, the maximum response of all blades when $\sigma$ is increasing is equal to 0.9978 while when $\sigma$ is decreasing, the maximum response is equal to 0.5766 . However, for mistuned system, the maximum response of each blade is different and the blade with the highest mistuning has the largest maximum response. For our case study, the most mistuned blade is the first one and this blade has the largest maximum response.

\subsection{Twelve-degree-of freedom system}

For a system with twelve blades, nonlinear algebraic system of equations was numerically calculated using a code developed in Matlab environment. In this example, the grounding stiffness parameter, $\hat{K}_{n}$ for all blades was set to 14 (i.e. $\hat{K}_{1}=\ldots=\hat{K}_{12}=14$ ). For the mistuned case, these parameters were selected randomly in the range of 4 to 24; these values are given in Table 3 .

Again responses of all twelve blades are plotted in Figure 6 for forward and backward system excitation, by solving Equations (28) and (29) for the case of 12DOFs system.

It can be seen from figures that the first jump for all blades almost happen in a specific frequency. The first jump frequency for increasing and decreasing mistuning frequency are -22 and $-30 \mathrm{~Hz}$ respectively. However, the second jump in both case happen in a wide frequency range. Figure 7 shows the blade response of both tuned and mistuned systems for the 12 DOFs system with $\hat{\mu}=0$.

Table 4 tabulated the maximum response of the blades for both tuned and mistuned systems. Again, the maximum response for mistuned system is larger than the tuned one. For the tuned system the response of all blades are the same while for the mistuned system each blade has different response amplitude. In our example, the eleventh blade has the highest perturbation and the highest vibration response. 
E. Raeisi Estabragh et al.: Mechanics \& Industry 17, 409 (2016)

Table 3. Values of $\hat{K}_{n}$ for the twelve-degree-of freedom mistuned system.

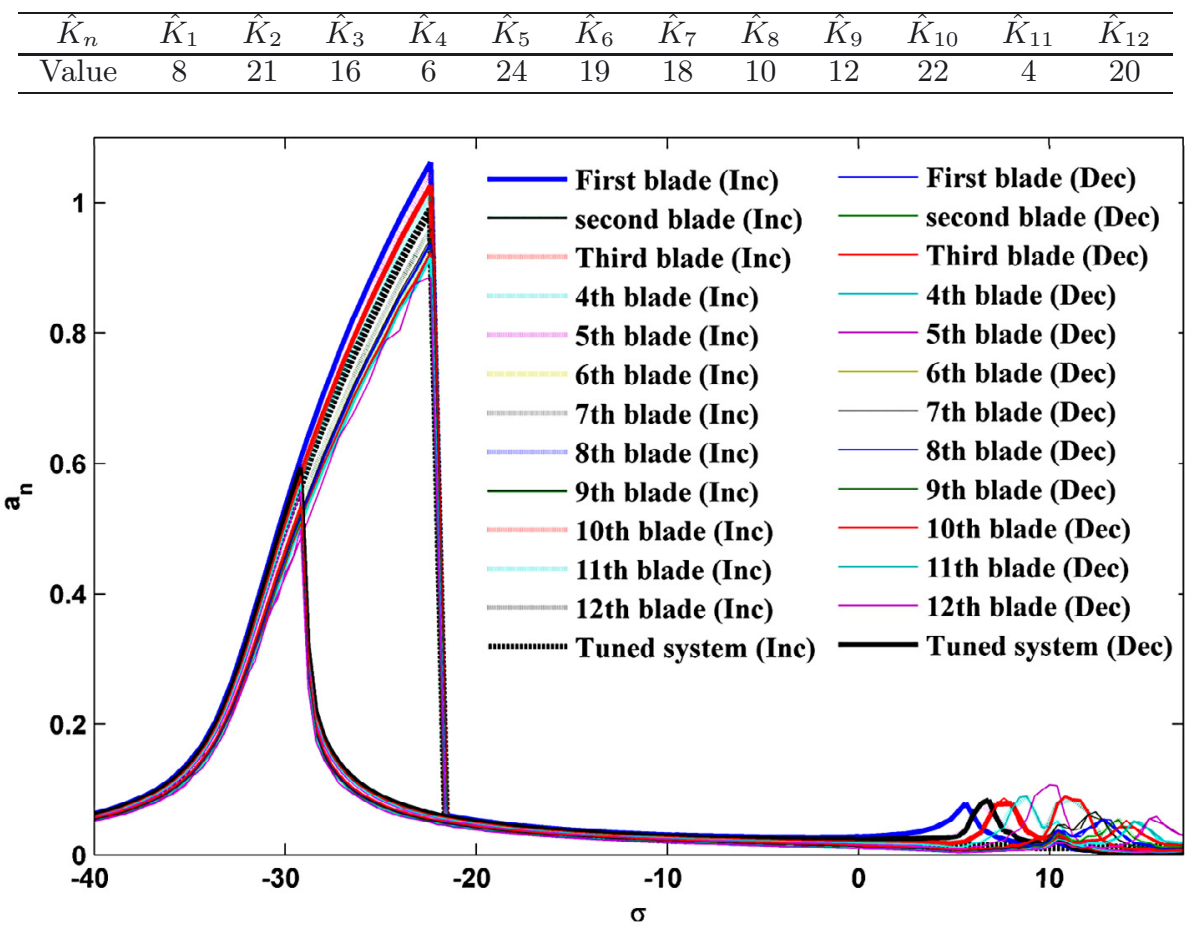

Fig. 6. Response amplitude, $a_{n}$, for all twelve mistuned pendulums.

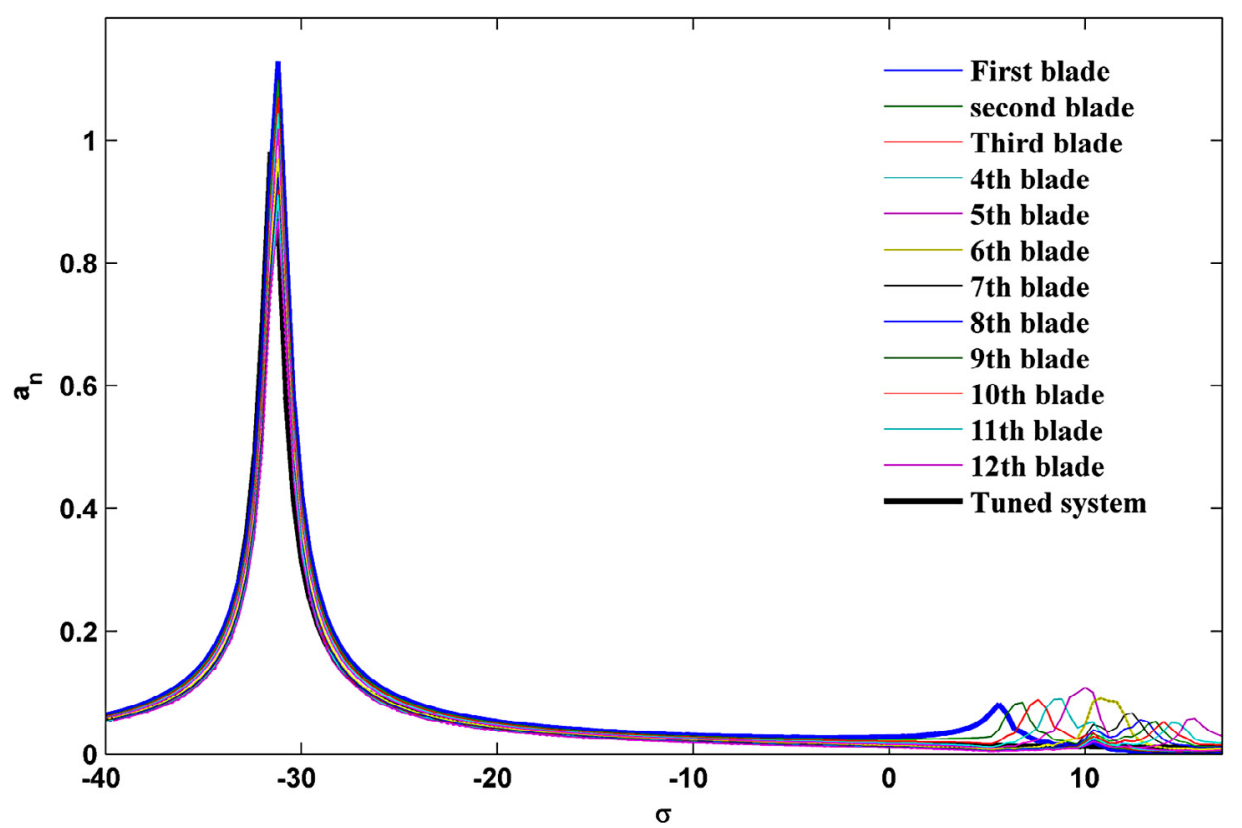

Fig. 7. Response amplitude, $a_{n}$, for all twelve mistuned pendulums (for $\hat{\mu}=0$ ).

\section{Conclusions}

Mistuning, imperfections in cyclical symmetry of bladed disks are an inevitable occurrence due to many factors including manufacturing tolerances and in service wear and tear. It can cause some unpredictable phenomena such as mode splitting, mode localization and dramatic difference in forced vibration response.
In order to investigate the effects of mistuning on nonlinear cyclical symmetric systems, the method of multiple scales is used for finding the forced and steady state vibration response of a nonlinear mistuned lumped model. For tuned system, all blades experience jump phenomenon at the same time and with equal amplitude. In addition, the results show that only one jump will happen for the tuned system during the excitation. The results indicate 


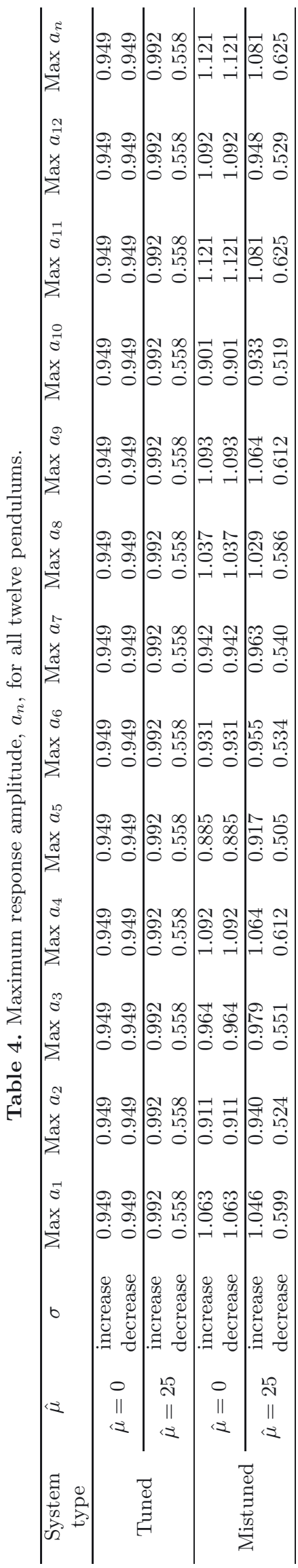

that jump phenomenon occurs several times during the forward and backward excitation of the mistuned nonlinear system. Moreover, the amplitude of vibration for each blade is different.

\section{References}

[1] A.H. Nayfeh, Nonlinear oscillations, Wiley, New York, 1995

[2] S.H. Strogatz, Nonlinear Dynamics and Chaos: with Applications to Physics, Biology, Chemistry, and Engineering, Addison-wesley, Reading, MA, 1994

[3] F. Verhulst, Nonlinear Differential Equations and Dynamical Systems, 2nd edition, Springer, Berlin, 1999

[4] R. Rand, Lecture Notes on Nonlinear Vibrations, Cornell, New York, USA, 2003

[5] R.M. Rosenberg, On nonlinear vibrations of systems with many degrees of freedom, Adv. Appl. Mech. 9 (1966) 155242

[6] R.H. Rand, C.H. Pak, A.F. Vakakis , Bifurcation of nonlinear normal modes in a class of two degree of freedom systems, Acta Mechanica 3 (1992) 129-145

[7] A.F. Vakakis, T. Nayfeh, M.E. King, A multiple scales analysis of nonlinear, localized modes in a cyclic periodic system, J. Appl. Mech. 60 (1993) 388-397

[8] M.E. King, A.F. Vakakis, Mode localization in a system of coupled flexible beams with geometric nonlinearities, Zeit. Angew. Math. Mech. (ZAMM) 75 (1995) 127-139

[9] A.F. Vakakis, L.I. Manevitch, Y.V. Mikhlin, V.N. Pilichuk, A.A. Zevin, Normal Modes and Localization in Nonlinear Systems, Wiley, New York, 1996

[10] R. Rand, A direct method for nonlinear normal modes, Int. J. Non-Linear Mech. 9 (1974) 363-368

[11] S.W. Shaw, C. Pierre, Normal modes for non-linear vibratory systems, J. Sound Vib. 164 (1993) 85-124

[12] A.F. Vakakis, Non-linear normal modes and their applications in vibration theory: an overview, Mech. Syst. Signal Process. 11 (1997) 3-22

[13] A.H. Nayfeh, Introduction to Perturbation Techniques, Wiley-Interscience, New York, 1981

[14] R.E. O'Malley, Singular Perturbation Methods for Ordinary Differential Equations, Springer, New York, 1991

[15] J. Kevorkian, J.D. Cole, Multiple Scales and Singular Perturbation Methods, Springer, New York, 1996

[16] H.S.Y. Chan, K.W. Chung, Z. Xu, A perturbationincremental method for strongly non-linear oscillators, Int. J. Non-Linear Mech. 31 (1996) 59-72

[17] S.H. Chen, Y.K. Cheung, A modified Lindstedt-Poincare' method for a strongly nonlinear two degree-of-freedom system, J. Sound Vib. 193 (1996) 751-762

[18] V.N. Pilipchuk, The calculation of strongly nonlinear systems close to vibration-impact systems, PMM 49 (1985) $572-578$

[19] L.I. Manevitch, Complex Representation of Dynamics of Coupled Oscillators in Mathematical Models of Nonlinear Excitations, Transfer Dynamics and Control in Condensed Systems, Kluwer Academic/Plenum Publishers, New York, 1999

[20] M.I. Qaisi, A.W. Kilani, A power-series solution for a strongly non-linear two-degree-of-freedom system, J. Sound Vib. 233 (2000) 489-494 
[21] V.I. Babitsky, V.L. Krupenin, Vibrations of Strongly Nonlinear Discontinuous Systems, Springer, Berlin, 2001

[22] M.A. Rotea, F.J. D'Amato, Efficient algorithms for mistuning analysis, in: Proceedings of the 15th triennial world congress, Barcelona, Spain, 2003

[23] M. Rahimi, S. Ziaei-Rad, Uncertainty treatment in forced response calculation of mistuned bladed disk, Math. Comput. Simul. 80 (2010) 1746-1757

[24] B.J. Olson, S.W. Shaw, Vibration absorbers for a rotating flexible structure with cyclic symmetry: nonlinear path design, Nonlinear Dynamics (2009) DOI: 10.1007/s11071-009-9587-8

[25] H.H. Yoo, J.Y. Kim, D.J. Inman, Vibration localization of simplified mistuned cyclic structures undertaking external harmonic force, J. Sound Vib. 261 (2003) 859-870

[26] M.E. King, Philip A. Layne , Dynamics of Nonlinear Cyclic Systems with Structural Irregularity, Nonlinear Dynamics 15 (1998) 225-244

[27] O. Gendelman, D. Gorlov, L. Manevitch, A. Musienko, Dynamics of coupled linear and essentially nonlinear oscillators with substantially different masses, J. Sound Vib. 286 (2005) 1-19

[28] Y.V. Mikhlin, S. Reshetnikova, Dynamical interaction of an elastic system and essentially nonlinear absorber, J. Sound Vib. 283 (2005) 91-120

[29] A.F. Vakakis, R.H. Rand, Non-linear dynamics of a system of coupled oscillators with essential stiffness nonlinearities, Int. J. Non-Linear Mech. 39 (2004) 1079-1091

[30] A.F. Vakakis, L.I. Manevitch, O. Gendelman, L. Bergman, Dynamics of linear discrete systems connected to local, essentially non-linear attachments, J. Sound Vib. 264 (2003) 559-577

[31] D.M. McFarland, L.A. Bergman, A.F. Vakakis, Experimental study of non-linear energy pumping occurring at a single fast frequency, Int. J. Non-Linear Mech. 40 (2005) 891-899

[32] S. Pernot, E. Gourdon, C. Lamarque, M. Gloeckner, T. Griessmann, Experimental dynamics of a four-storey building coupled with a nonlinear energy sink, in: ENOC, Eindhoven, Netherlands, 2005

[33] R.M. Rosenberg, The normal modes of nonlinear ndegrees-of-freedom systems, J. Appl. Mech. 30 (1962) 595-611

[34] O.V. Gendelman, Bifurcations of nonlinear normal modes of linear oscillator with strongly nonlinear damped attachment, Nonlinear Dynamics 37 (2004) 115-128

[35] F.X. Wang, A.K. Bajaj, Nonlinear normal modes in multi-mode models of an inertially coupled elastic structure, Nonlinear Dynamics 47 (2007) 25-47

[36] W. Lacarbonara, G. Rega, A.H. Nayfeh, Resonant nonlinear normal modes, Part I: analytical treatment for structural one-dimensional systems, Int. J. Non-Linear Mech. 38 (2003) 851-872

[37] W. Lacarbonara, G. Rega, Resonant non-linear normal modes, Part II: activation/orthogonality conditions for shallow structural systems, Int. J. Non-Linear Mech. 38 (2003) 873-887
[38] C.E.N. Mazzilli, O.G.P. Baracho Neto, Evaluation of nonlinear normal modes for finite-element models, Comput. Struct. 80 (2002) 957-965

[39] X. Li, J.C. Ji, C.H. Hansen, Non-linear normal modes and their bifurcation of a two DOF system with quadratic and cubic non-linearity, Int. J. Non-linear Mech. 41 (2006) $1028-1038$

[40] A. Grolet, F. Thouverez, Vibration analysis of a nonlinear system with cyclic symmetry, J. Eng. Gas Turbines Power 133 (2011) 022502

[41] F. Georgiades, M. Peeters, G. Kerschen, J. Golinval, Modal analysis of a nonlinear periodic structure with cyclic symmetry, AIAA J. 47 (2009) 1014-1025

[42] S. Samaranayake, G. Samaranayake, A.K. Bajaj, Resonant vibrations in harmonically excited weakly coupled mechanical systems with cyclic symmetry, Chaos, Solitons and Fractals 11 (2000) 1519-1534

[43] W. Sextro, K. Popp, T. Krzyzynski, Localization in Nonlinear Mistuned Systems with Cyclic Symmetry, Nonlinear Dynamics 25 (2001) 207-220

[44] J. Judge, C. Pierre, O. Mehmed, Experimental Investigation of Mode Localization and Forced Response Amplitude Magnification for a Mistuned Bladed Disk, J. Eng. Gas Turbines Power 123 (2001) 940-950

[45] E.P. Petrov, D.J. Ewins, Analytical Formulation of Friction Interface Elements for Analysis of Nonlinear MultiHarmonics Vibrations of Bladed Disks, ASME J. Turbomachinery 125 (2003) 364371

[46] E.P. Petrov, D.J. Ewins, Method for Analysis of Nonlinear Multiharmonic Vibrations of Mistuned Bladed Disks With Scatter of Contact Interface Characteristics. ASME J. Turbomachinery 127 (2006) 128136

[47] E. Ciğeroğlu, H.N. Özgüven, Nonlinear vibration analysis of bladed disks with dry friction dampers, J. Sound Vib. 292 (2006) 10281043

[48] Y.J. Yan, P.L. Cui, H.N. Hao, Vibration mechanism of a mistuned bladed-disk, J. Sound Vib. 317 (2008) 294-307

[49] B.K. Beachkofski, Probabilistic rotor life assessment using reduced order models, J. Shock Vib. 16 (2009) 581591

[50] D. Laxalde, F. Thouverez, J.J. Sinou, J.P. Lombard, Qualitative analysis of forced response of blisks with friction ring dampers, Eur. J. Mech. A/Solids 26 (2007) 676687

[51] S.H. Shin, M.K. Kang, H.H. Yoo, Mistuned coupling stiffness effect on the vibration localization of cyclic systems, J. Mech. Sci. Technol. 22 (2008) 269-275

[52] B. Salhi, J. Lardies, M. Berthillier, Identification of modal parameters and aeroelastic coefficients in bladed disk assemblies, Mech. Syst. Signal Process. 23 (2009) 1894-1908

[53] B. Zhou, F. Thouverez, D. Lenoir, An adaptive control strategy based on passive piezoelectric shunt techniques applied to mistuned bladed disks, J. Comput. Appl. Math. 246 (2013) 289-300

[54] B. Zhou, F. Thouverez, D. Lenoir, Essentially nonlinear piezoelectric shunt circuits applied to mistuned bladed disks, J. Sound Vib. 333 (2014) 2520-2542 